\title{
Development and Verification of an Immune-Related Gene Pairs Prognostic Signature in Hepatocellular Carcinoma
}

\section{OPEN ACCESS}

Edited by:

Ismail Hosen,

University of Dhaka, Bangladesh

Reviewed by:

Giuliana Cavalloni,

Fondazione del Piemonte per

l'Oncologia (IRCCS), Italy

Udhaya Kumar S,

Vellore Institute of Technology, India

Sanjay Mishra,

The Ohio State University,

United States

Harpreet Kaur,

Pine Biotech, United States

${ }^{*}$ Correspondence:

Wenji Wang

Idyyjzwwj@163.com

${ }^{t}$ These authors have contributed equally to this work and share first

authorship.

Specialty section: This article was submitted to

Molecular Diagnostics and Therapeutics,

a section of the journal

Frontiers in Molecular Biosciences

Received: 27 May 2021 Accepted: 21 September 2021 Published: 01 October 2021

Citation:

Feng $X$, MU S, Ma Y and Wang W (2021) Development and Verification of an Immune-Related Gene Pairs Prognostic Signature in

Hepatocellular Carcinoma.

Front. Mol. Biosci. 8:715728.

doi: $10.3389 /$ /fmolb.2021.715728

\author{
Xiaofei Feng ${ }^{1 \dagger}$, Shanshan $\mathrm{Mu}^{2 \dagger}$, Yao $\mathrm{Ma}^{3 \dagger}$ and Wenji Wang ${ }^{1 *}$ \\ ${ }^{1}$ Department of Orthopedics, Lanzhou University First Affiliated Hospital, Lanzhou, China, ${ }^{2}$ Pediatric Rheumatism Immunology \\ Department, Lanzhou University Second Hospital, Lanzhou, China, ${ }^{3}$ Clinical Laboratory Center, Gansu Provincial Maternity and \\ Child-care Hospital, Lanzhou, China
}

With the increasing prevalence of Hepatocellular carcinoma $(\mathrm{HCC})$ and the poor prognosis of immunotherapy, reliable immune-related gene pairs (IRGPs) prognostic signature is required for personalized management and treatment of patients. Gene expression profiles and clinical information of HCC patients were obtained from the TCGA and ICGC databases. The IRGPs are constructed using immune-related genes (IRGs) with large variations. The least absolute shrinkage and selection operator (LASSO) regression analysis was used to construct IRGPs signature. The IRGPs signature was verified through the ICGC cohort. 1,309 IRGPs were constructed from 90 IRGs with high variability. We obtained 50 IRGPs that were significantly connected to the prognosis and constructed a signature that included 17 IRGPs. In the TCGA and ICGC cohorts, patients were divided into high and low-risk patients by the IRGPs signature. The overall survival time of low-risk patients is longer than that of high-risk patients. After adjustment for clinical and pathological factors, multivariate analysis showed that the IRGPs signature is an independent prognostic factor. The Receiver operating characteristic (ROC) curve confirmed the accuracy of the signature. Besides, gene set enrichment analysis (GSEA) revealed that the signature is related to immune biological processes, and the immune microenvironment status is distinct in different risk patients. The proposed IRGPs signature can effectively assess the overall survival of HCC, and provide the relationship between the signature and the reactivity of immune checkpoint therapy and the sensitivity of targeted drugs, thereby providing new ideas for the diagnosis and treatment of the disease.

\footnotetext{
Keywords: hepatocellular carcinoma, immune-related gene pairs, prognosis, tumor immune environment, targeting therapy

Abbreviations: AUC, area under curve; GO, gene ontology; GSEA, gene set enrichment analysis; HCC, hepatocellular carcinoma; HR, hazard ratio; ICGC, international cancer genome consortium; IRG(s), immune-related gene(s); IRGP(s), immune-related gene pair(s); KEGG, kyoto encyclopedia of genes and genomes; LASSO, least absolute shrinkage and selection operator; PCA, principal component analysis; ROC, receiver operating characteristic; TCGA, the cancer genome atlas.
} 


\section{INTRODUCTION}

Hepatocellular carcinoma (HCC) is one of the most common malignant tumors and the leading cause of cancer-related deaths, and its morbidity and mortality are increasing (Forner et al., 2018; Villanueva, 2019). According to the latest cancer statistics in 2019, approximately 42,030 people in the United States are diagnosed with liver cancer each year, and 31,780 people die from the disease (Siegel et al., 2019). Although breakthroughs in the diagnosis and treatment of HCC have been made in recent years, the prognostic outcome of patients remains poor (Yang et al., 2019). The current clinical application of immunotherapy in HCC has benefited some patients, but this approach has not effectively improved the prognosis, and the long-term survival rate is still very poor (Dong et al., 2020). Due to the complexity and heterogeneity of HCC, individualized decision-making plans are required in diagnosis and treatment. Therefore, it is necessary to identify the novel prognostic signature of HCC and use it to guide clinical treatment as an effective way to improve the prognosis of patients.

Understanding the characteristics of tumor immune cell infiltration can improve the responsiveness of immunotherapy and is of great help in understanding the mechanism of cancer occurrence (Ino et al., 2013; Binnewies et al., 2018). The landscape of immune cells in HCC mapped by single-cell sequencing and other evidence indicate that the tumor immune microenvironment plays an indispensable role in the progression of HCC (Nishida and Kudo, 2017; Zheng et al., 2017; Kurebayashi et al., 2018). Different immune cells infiltrating into HCC have different prognostic effects (Prieto et al., 2015; Cariani and Missale, 2019). It has been observed clinically that the increase of $\mathrm{PD}^{+} \mathrm{CD}^{+} \mathrm{T}$ cells in HCC is associated with poor clinical outcomes (Chew et al., 2017). Although numerous studies have found the importance of immunology in HCC, its molecular mechanism is still unclear. Current studies have shown that tumor immune-related markers have commendable effects on the diagnosis and treatment of cancer (Peng et al., 2019; Hong et al., 2020). There have been many studies on the prognostic value of identifying key genes to build models to predict the prognosis of HCC patients (Li et al., 2017a; Kaur et al., 2019; Liu et al., 2020; Ouyang et al., 2020). However, there is no in-depth survey on the clinical relevance and prognostic significance of IRGPs in HCC.

In this study, we used the HCC gene expression datasets of the TCGA and ICGC databases to develop individualized prognostic signature based on IRGPs. Then, we evaluated its ability to predict prognosis in HCC patients and its responsiveness to immune checkpoint therapy and targeted therapy.

\section{MATERIALS AND METHODS}

\section{Data Sources}

The HCC level 3 RNA-seq data and clinical information were downloaded from the TCGA database, including normal tissues $(n=50)$ and tumor tissues $(n=374) .365$ cases of tumor patients have survival time and survival status, of which 247 cases contain clinical information (age, gender, histologic grade, TNM stage, vascular invasion, and alpha fetoprotein). The RNA-seq data and clinical information of HCC were downloaded from the ICGC database, including normal tissues $(n=202)$ and tumor tissues $(n=243) .232$ tumor patients have survival time, survival status, and clinical information (age, gender, and TNM stage). The list of IRGs was downloaded from the IMMPORT (Bhattacharya et al., 2018). Both the TCGA and ICGC data are publicly available. Therefore, this research does not require the approval of the local ethics committee.

\section{Construction of an Individualized Prognostic Signature Based on IRGPs}

In the TCGA and ICGC cohorts, the limma package (Ritchie et al., 2015) was used to identify the IRGs that are different between cancer tissues and normal tissues. The filtering criteria were $|\log \mathrm{FC}|>1$, FDR $<0.01$. We identified the common differential IRGs in the two cohorts. The protein-protein interaction network was used to demonstrate differential genes (Udhaya Kumar et al., 2021), and the Cytoscape software (v3.8.2) was used for visualization. Genes with the median absolute deviation greater than 1 in tumor samples whose expression levels of these common differential IRGs were considered as candidate genes. The expression levels of these candidate genes were compared in pairs to generate a score for each IRGP. If IRG 1 is less than IRG 2, the IRGP score is 1, otherwise, the IRGP score is 0 ( $\mathrm{Li}$ et al., 2017b). In the TCGA cohort, univariate cox was used to select prognostic IRGPs to assess the association between each IRGP and the overall survival rate of the patient. Prognostic IRGPs with a $p$ value of less than 0.001 were candidates for establishing an IRGPs signature. From these IRGPs, the $\mathrm{R}$ language was used to perform Lasso Cox proportional hazard regression to construct the risk score, and finally 17 IRGPs were used to define the IRGPs signature. To classify patients into highor low-risk patients, the optimal IRGPs signature cut-off value was determined by the ROC curve.

\section{Verification of IRGPs Signature}

In the TCGA and ICGC cohorts, the survival package and survminer package (https://CRAN.R-project.org/package = survminer) were used to establish the survival curve of the high and low-risk patients through the Kaplan-Meier diagram, and the Log-rank test was used to analyze the difference in survival curves. Cox proportional hazards analysis was used for univariate and multivariate analysis. The survivalROC package (https://CRAN.R-project.org/package = survivalROC) was used for ROC curve analysis to evaluate the predictive ability of the IRGPs signature.

\section{Functional Annotation and Immune Cell Infiltration}

To understand the underlying molecular mechanism of the IRGPs signature, we divided the TCGA cohort patients into high- and low-risk patients so that the software GSEA 4.1.0 (http://www.gsea-msigdb.org) can be used for gene ontology 

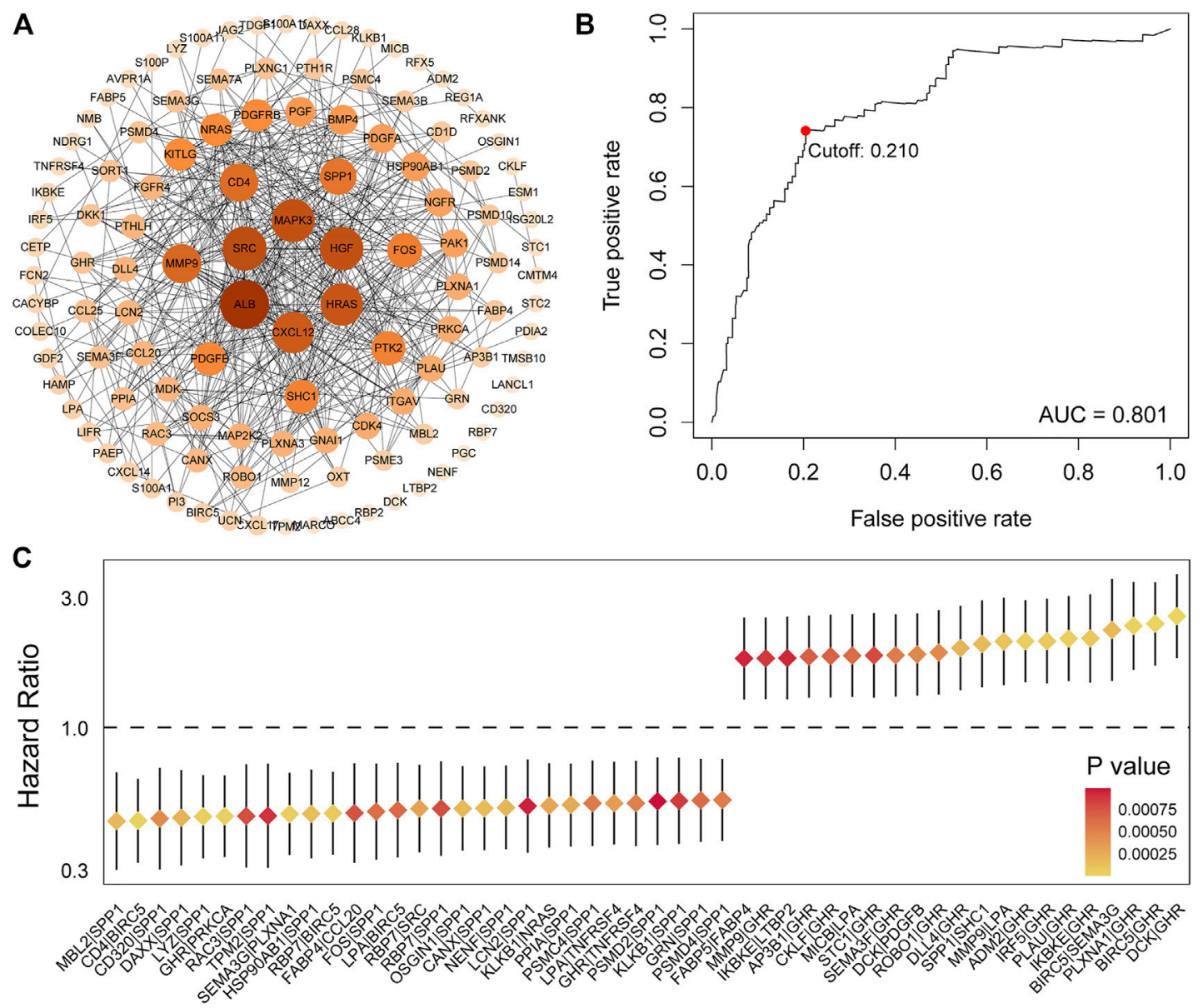

FIGURE 1 | Identification of IRGs and determination of risk score. (A) 120 shared differential immunity related genes were displayed on the protein-protein interaction network. (B) Univariate Cox analysis obtained 50 IRGPs related to prognosis. (C) ROC curve of the risk score of the IRGPs signature in the TCGA cohort. The risk score was 0.210 , which served as a threshold for classifying patients into the high and low-risk patients.

(GO) and Kyoto Encyclopedia of Genes and Genomes (KEGG) annotations. When $p<0.05$ and FDR $<0.25$, the enriched gene set is considered to be statistically significant. The gsva package (Hänzelmann et al., 2013) was used to quantify immune cell infiltrations, and the correlation between high- and low-risk patients and 16 immune cell infiltrations and 13 immunerelated pathways was evaluated.

\section{Explore the Relationship Between the IRGPs Signature and the Reactivity of Immune Checkpoint Therapy and the Sensitivity of Targeted Drugs}

Immune checkpoint blockers of immunotherapy have obvious effects in the treatment of many human cancers. Therefore, we explored the expression and correlation between high and lowrisk patients and the current common immune checkpoints. In addition, small molecule tyrosine kinase inhibitors, as a class of molecularly targeted drugs, have become one of the mainstream trends in current anti-liver cancer research. The pRRophetic package is used to analyze the sensitivity of tyrosine kinase inhibitors in high- and low-risk patients (Geeleher et al., 2014).

\section{RESULTS}

\section{Construction and Definition of the IRGPS Signature}

Principal component analysis (PCA) was first performed on the expression profiles of the two cohorts. The results showed that there were differences in the distribution of tumor tissue and normal tissue in the two data sets, which could be used for further analysis (Supplementary Figures S1A,C). 231 differential IRGs were identified in the TCGA cohort and 172 differential IRGs were identified in the ICGC cohort (Supplementary Figures S1B,D). We obtained 120 shared differential IRGs and displayed them in the protein-protein interaction network (Figure 1A). In order to obtain genes with high variability, we screened among 120 IRGs. The filter criterion was that the median absolute deviation of these genes in tumor samples is greater than 1.90 
TABLE 1 | Baseline characteristics of the patients in different risk groups.

\begin{tabular}{|c|c|c|c|c|c|c|c|c|}
\hline \multirow[t]{2}{*}{ Variables } & \multicolumn{4}{|c|}{ TCGA cohort } & \multicolumn{4}{|c|}{ ICGC cohort } \\
\hline & High risk & low risk & $x^{2}$ & $\mathbf{P}$ & High risk & low risk & $x^{2}$ & $\mathbf{P}$ \\
\hline Age (year) & - & - & 0 & 1 & - & - & 150.09 & $<0.001$ \\
\hline$<60$ & 50 (50.0\%) & 119 (46.3\%) & - & - & 5 (16.4\%) & 36 (23.6\%) & - & - \\
\hline$\geqq 60$ & 58 (50.0\%) & 138 (53.7\%) & - & - & 58 (83.6\%) & 129 (76.4\%) & - & - \\
\hline Gender & - & - & 0.1 & 0.75 & - & - & 5.13 & 0.02 \\
\hline female & 37 (34.3\%) & 82 (31.9\%) & - & - & 25 (37.3\%) & 36 (21.8\%) & - & - \\
\hline male & 71 (65.7\%) & 175 (68.1\%) & - & - & 42 (62.7\%) & 129 (78.2\%) & - & - \\
\hline Histologic grade & - & - & 19.71 & $<0.001$ & - & - & - & - \\
\hline G1 and G2 & 50 (46.3\%) & 180 (70.0\%) & - & - & - & - & - & - \\
\hline G3 and G4 & 57 (52.8\%) & 73 (28.4\%) & - & - & - & - & - & - \\
\hline unknow & 1 (0.9\%) & $4(1.6 \%)$ & & & - & - & - & - \\
\hline Child-Pugh grade & & - & 6.49 & 0.04 & - & - & - & - \\
\hline A & 53 (49.1\%) & 163 (63.4\%) & - & - & - & - & - & - \\
\hline $\mathrm{B}$ and $\mathrm{C}$ & 8 (7.4\%) & $14(5.4 \%)$ & - & - & - & - & - & - \\
\hline unknow & 47 (43.5\%) & 80 (31.1\%) & - & - & - & - & - & - \\
\hline Alpha fetoprotein & - & - & 15.69 & $<0.001$ & - & - & - & - \\
\hline$<200$ ng/ml & 43 (39.8\%) & 158 (61.5\%) & - & - & - & - & - & - \\
\hline$\geqq 200 \mathrm{ng} / \mathrm{ml}$ & 33 (30.6\%) & 42 (16.3\%) & - & - & - & - & - & - \\
\hline unknow & 32 (29.6\%) & 57 (22.2\%) & - & - & - & - & - & - \\
\hline Vascular invasion & - & - & 20.46 & $<0.001$ & - & - & - & - \\
\hline YES & 40 (37.0\%) & 66 (25.7\%) & - & - & - & - & - & - \\
\hline NO & 42 (38.9\%) & 163 (63.4\%) & - & - & - & - & - & - \\
\hline unknow & 26 (24.1\%) & 28 (10.9\%) & - & - & - & - & & - \\
\hline TNM stage & - & - & 13.57 & $<0.001$ & - & - & 9.76 & $<0.001$ \\
\hline | and || & 65 (60.2\%) & 189 (73.5\%) & - & - & 30 (44.8\%) & 112 (67.9\%) & - & - \\
\hline III and IV & 39 (36.1\%) & 48 (18.7\%) & - & - & 37 (55.2\%) & 53 (32.1\%) & - & - \\
\hline unknow & $4(3.7 \%)$ & 20 (7.8\%) & - & - & - & - & - & - \\
\hline
\end{tabular}

IRGs were finally got for constructing 1,309 pairs of IRGPs. The correlation between these IRGPs and overall survival was evaluated in the TCGA cohort, and 50 IRGPs related to prognosis were screened out, with $p<0.001$ as the cut-off criterion (Figure 1C). A signature composed of 17 IRGPs was constructed on the TCGA cohort through LASSO Cox proportional hazard regression. The IRGPs signature consists of 22 unique IRGs (Supplementary Table S1). Finally, the timedependent ROC curve was used to determine the optimal cutoff value of 0.210 for the risk score, classified patients as high- or lowrisk patients (Figure 1B). At this point, the sensitivity was $74.1 \%$ and the specificity was $75.5 \%$. In the TCGA cohort, the high-risk patients were found to be significantly correlated with histologic grade, Child-Pugh grade, alpha-fetoprotein, vascular invasion, and TNM stage (Table 1). Overall survival in the TCGA cohort was worse in the high-risk patients than in the low-risk patients (Log-rank, $p<0.001$ ) (Figure 2A). PCA analysis shows that the distribution patterns of patients in different risk patients are contrasting (Figure 2B). The ROC curve assessed the prognostic ability of the prognostic signature, and the area under the curve (AUC) reaches 0.809 at 1 year, 0.757 at 2 years, and 0.712 at 3 years (Figure 2C).

\section{Validation of the Feasibility of the IRGPs Signature to Predict Survival}

To determine whether the signature has prognostic value, the signature was applied to the ICGC cohort as independent external verification. Patients in the ICGC cohort were divided into high or low-risk patients based on the above risk score. The high-risk patients in the ICGC cohort were also correlated with the TNM stage (Table 1). Also, the overall survival rate of the high-risk patients in the ICGC cohort is lower than in the low-risk patients (Log-rank, $p<0.001$ ) (Figure 2D). PCA analysis showed that the distribution pattern of the two groups of patients was distinct (Figure 2E). In addition, the ROC curve showed 0.772 at 1 year, 0.744 at 2 years, and 0.759 at 3 years (Figure 2F). These results are similar to those obtained in the TCGA cohort.

\section{Validation of the IRGPs Signature as an Independent Prognostic Factor}

In the TCGA cohort, univariate COX analysis showed that TNM stage $(p=0.002)$, vascular invasion $(p=0.035)$, and the IRGPs signature $(p<0.001)$ were significantly correlated with the prognosis of HCC. After adjusting for clinical and pathological factors such as age, gender, histologic grade, TNM stage, vascular invasion, and alpha-fetoprotein, the TNM stage (HR, 2.098; 95\% CI, 1.226,3.590; $p=0.007$ ) and the IRGPs signature (HR, 3.688; 95\%CI, 2.222,6.119; $p<0.001)$ were independent risk factors in the multivariate COX analysis (Figure 3A). Similarly, in the ICGC cohort, univariate COX analysis showed that TNM stage $(p<0.001)$ and the IRGPs signature $(p<0.001)$ were also significantly correlated with the prognosis of hepatocellular carcinoma. Multivariate COX analysis revealed that the TNM stage (HR, 1.882; 95\%CI, 1.319,2.685; $p<0.001)$ and the IRGPs signature (HR, 4.340; 95\% CI, 2.272, 8.291; $p<0.001)$ were also independent risk factors of overall survival (Figure $3 \mathbf{B}$ ). In the 

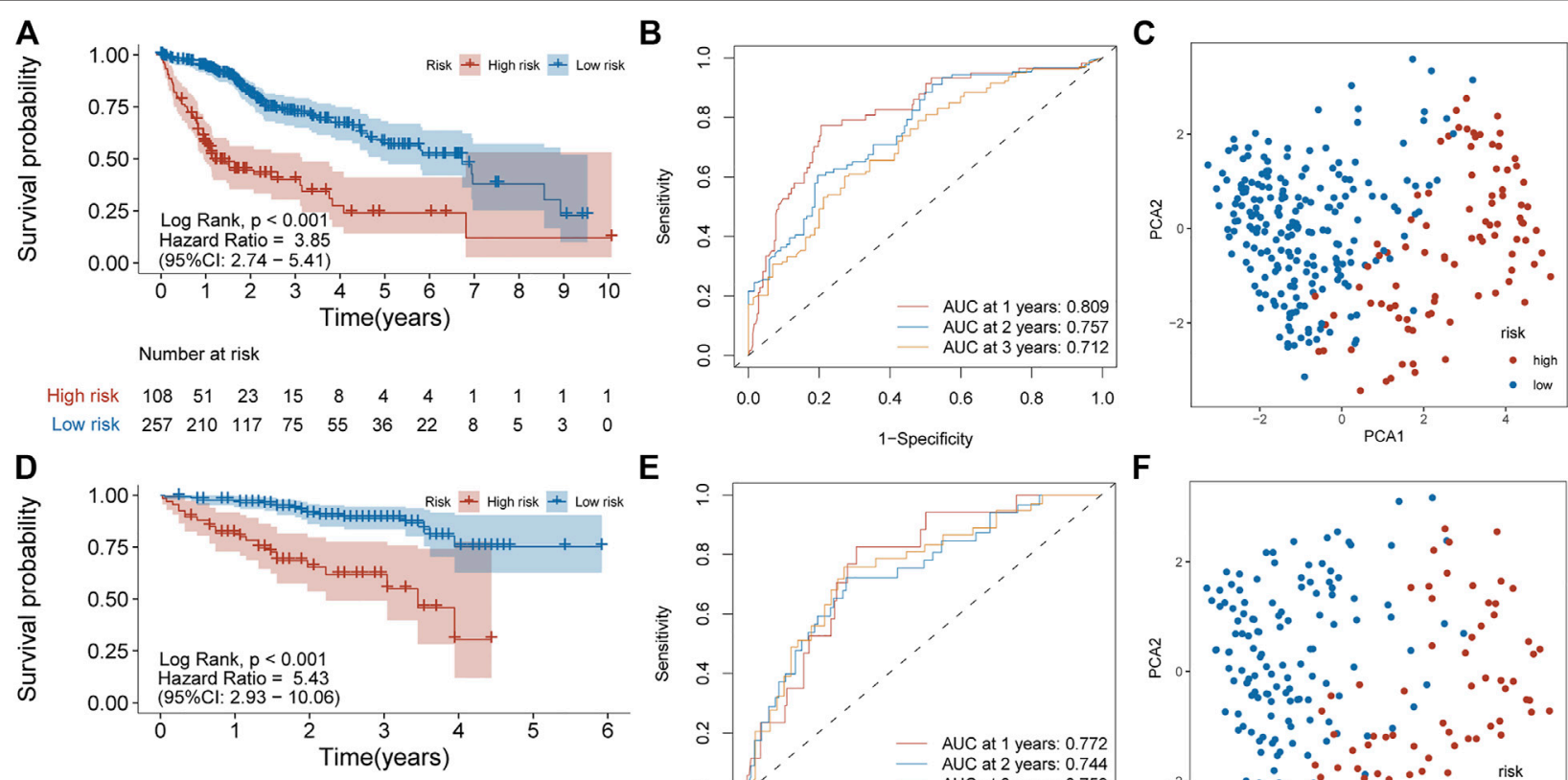

E
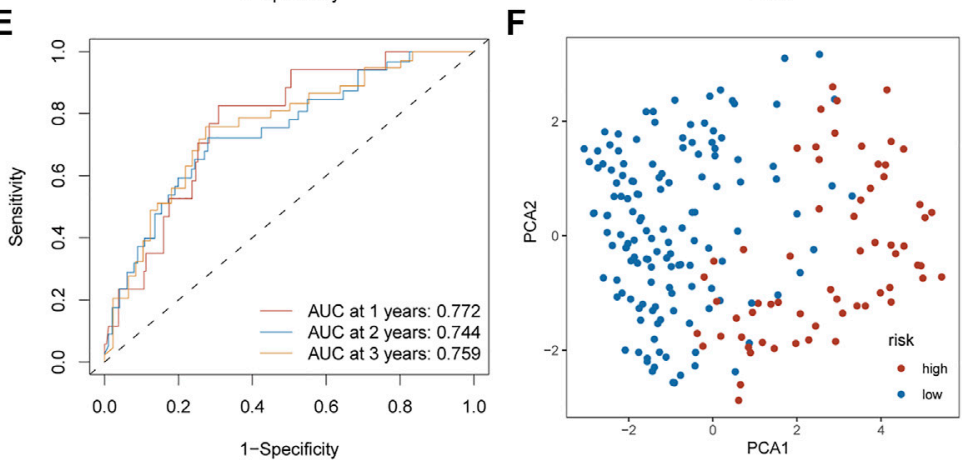

FIGURE 2|Prognostic analysis of the IRGPs signature in TCGA and ICGC cohorts. (A) Kaplan-Meier curve of overall survival in the high- and low-risk patients in the TCGA cohort. (B) ROC curve in TCGA cohort. (C) PCA plot of TCGA cohort. (D) Kaplan-Meier curve of overall survival in the high- and low-risk patients in the ICGC cohort. (E) ROC curve in ICGC cohort (F) PCA plot of ICGC cohort.

TCGA and ICGC cohorts, high and low-risk patients have different distributions in the TNM stage (Supplementary Figures S2A,C). In addition, the IRGPs signature divided patients with early (I and II) and late (III and IV) HCC into different prognostic groups. Also, for patients with stage IandII disease, low-risk patients have a good prognosis in both the TCGA cohort (Log-rank, $p<0.001$ ) and the ICGC cohort (Logrank, $p<0.001$ ) (Supplementary Figures S2B,D). Similarly, for patients with advanced stages III and IV, low-risk patients also have a good prognosis in the TCGA cohort (Log-rank, $p<0.001$ ) and ICGC cohort (Log-rank, $p=0.01$ ) (Supplementary Figures S2B,D). Overall, the IRGPs signature seems to be able to independently assess the overall survival of HCC.

\section{Functional Annotation and Immune Cell Infiltration Between High and Low-Risk Patients}

GSEA results showed that some immune-related biological processes are involved in high-risk patients (Supplementary Figure S3A), such as activation of innate immune response, antigen processing and presentation of peptide antigen via MHC class I, positive regulation of activated $\mathrm{T}$ cell proliferation, regulation of type I interferon mediated signalling pathway. Interestingly, some immune-related KEGG pathways are enriched in high-risk patients (Supplementary Figures S3B), such as FC epsilon RI signalling pathway, MAPK signalling pathway, mTOR signalling pathway, NOD like receptor signalling pathway. These results indicate that immune-related biological processes may play an indispensable role in the development of HCC. We further explored the status of immune cells and immune-related functions in high-risk and low-risk populations. In the TCGA cohort, the high-risk patients were positively correlated with tumor-infiltrating immune cells (aDCs, Macrophages, TH2 cells, Treg), while negatively correlated with Mast cells, Neutrophils, and NK cells (Figure 4A). In the ICGC cohort, except for Mast cells, which were not statistically significant, the results were consistent with the results in TCGA (Figure 4C). In the TCGA cohort, the highrisk patients were positively correlated with MHC class I and negatively correlated with Type II IFN Response (Figure 4B). In the ICGC cohort, the same result was obtained (Figure 4D).

\section{Reactivity of Immune Checkpoint Therapy and Sensitivity of Targeted Drugs}

Immune checkpoint therapy has shown better results in the treatment of cancer, and it has made a major breakthrough in the field of HCC. Therefore, we investigated the expression of immune checkpoint markers in low and high-risk patients. In the TCGA and ICGC cohorts, immune checkpoint markers (CD276, HHLA2, TNFRSF18, TNFSF9, LGALS9) were expressed higher in high-risk patients, and there was a positive correlation between the IRGPs signature and these markers $(|R|>0.3, p<0.05$, Figures 5A-E). Tyrosine kinase inhibitors currently approved and in clinical trials have demonstrated efficacy in the treatment 


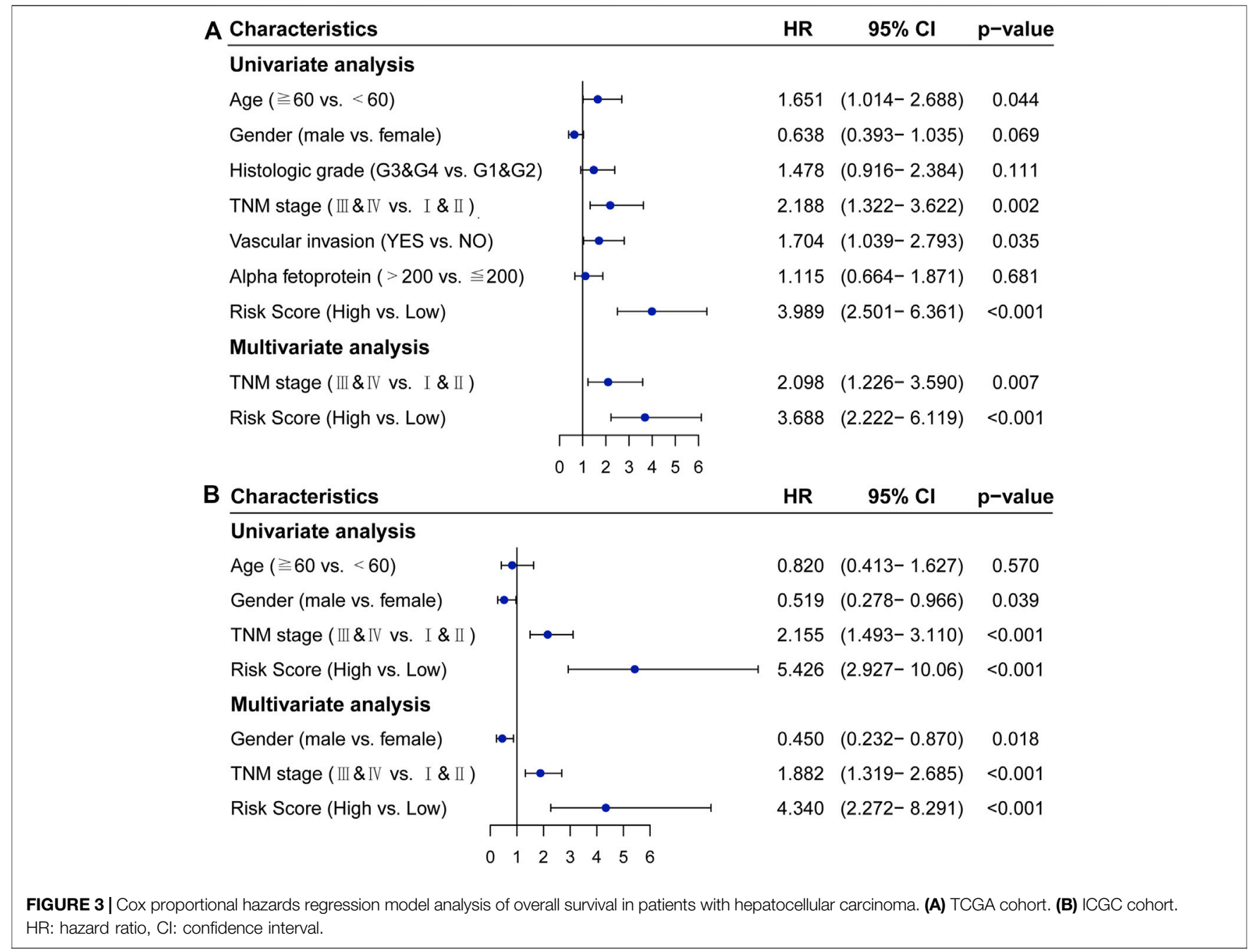

of hepatocellular carcinoma, which can be helpful in the treatment and management of patients with HCC. Hence, we evaluated the IC50 of each sample and observed that the IC50 of the eight small molecule tyrosine kinase inhibitors was significantly different between the two groups. The results showed that Axitinib, Motesanib, Dasatinib, Nilotinib, Erlotinib, Pazopanib, Lapatinib, and Saracatinib are more sensitive to low-risk patients $(p<0.001$, Figures $6 \mathbf{A}-\mathbf{H})$. This may provide an accurate strategy for the treatment of HCC patients.

\section{DISCUSSION}

Immune infiltration plays an important role in cancer progression. With the exploration in the field of immunotherapy, it is of great help in the treatment of tumor. There have been studies that have provided strong evidence for the treatment and diagnosis of some diseases through bioinformatics analysis (Kumar et al., 2019; Udhaya Kumar et al., 2020). Due to the high heterogeneity of HCC, some patients fail to achieve the expected curative effect on immunotherapy. Therefore, it is extremely crucial to determine the sensitivity of different patient subsets to treatment response. Individualized treatment for different patient subgroups will help to enhance the prognosis of patients (Gan et al., 2020; Zhou et al., 2020; Kaur et al., 2021). Novel signature related to tumor immune infiltration may be a sword for identifying new molecular targets and improving patient prognosis.

In this study, we developed a prognostic signature based on 17 IRGPs in HCC and validated them in two independent data sets on different platforms. Our prognostic immune signature can further divide clinically defined patients [for example, early stage (I and II) and late stage (III and IV)] into subgroups with different survival outcomes. Univariate COX analysis showed that TNM stage and the IRGPs signature were significantly correlated with the prognosis of HCC. By multivariate COX analysis, the TNM stage and the prognostic signature can be used as independent prognostic factors. Therefore, our prognostic signature can be used as a personalized prognosis and diagnosis and treatment of HCC patients and can be easily translated into clinical practice. 


\section{A Risk 白 low 白 nigh}

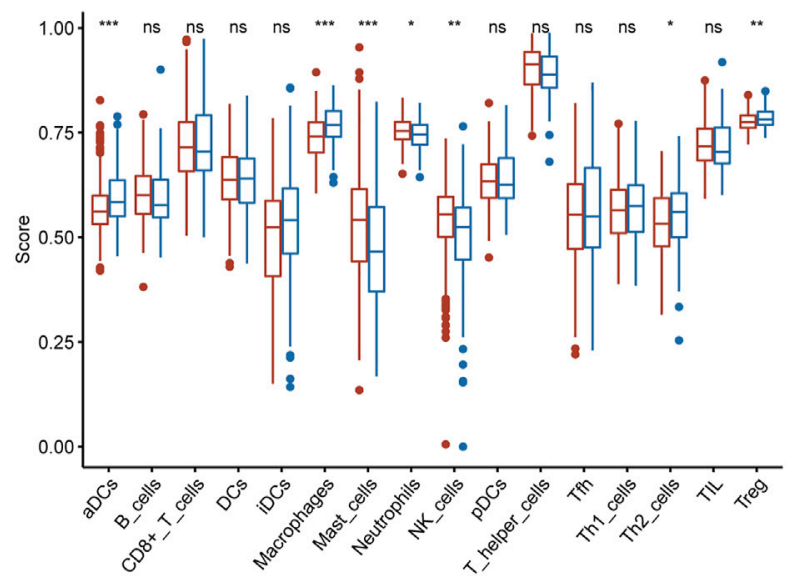

C

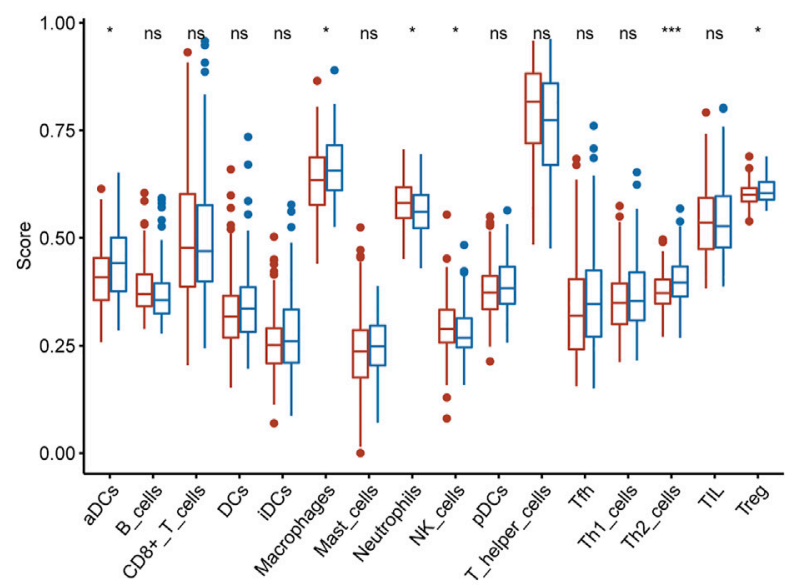

B

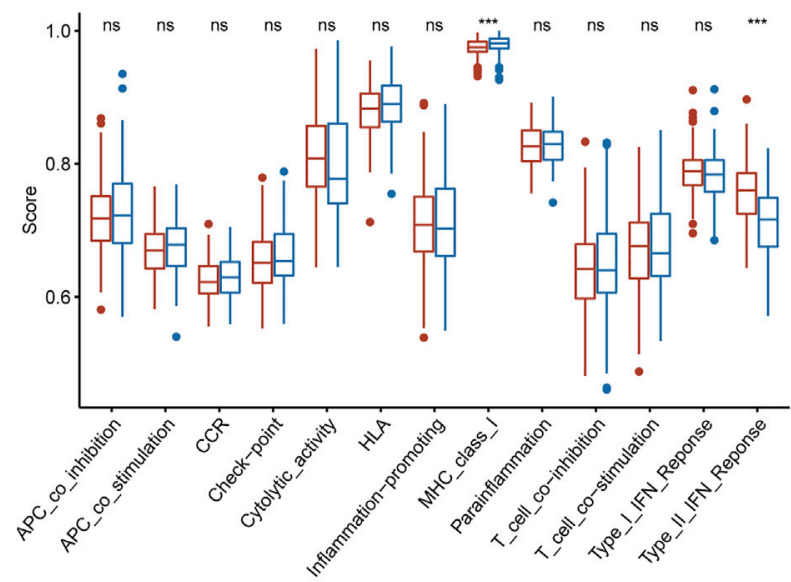

D

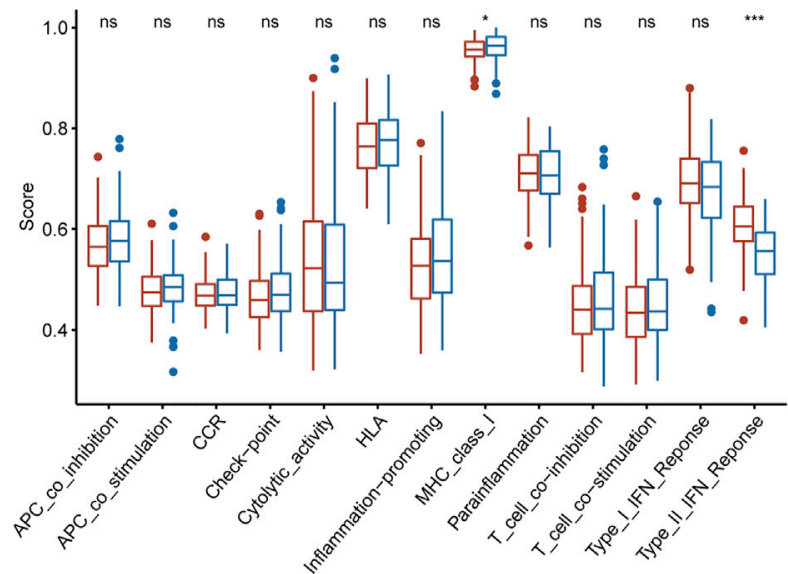

FIGURE 4 | The relationship between the IRGPS signature and immune infiltrating cells and immune-related functions. (A) The relationship between the IRGPS signature and immune cells in the TCGA cohort. (B) The relationship between the IRGPs signature and immune-related functions in the TCGA cohort. (C) The relationship between the IRGPs signature and immune cells in the ICGC cohort. (D) The relationship between the IRGPs signature and immune-related functions in the ICGC cohort. The adjusted $p$ value is ns, which is not significant. ${ }^{\star} p<0.05 ;{ }^{\star *} p<0.01 ;{ }^{\star \star *} p<0.001$.

The IRGPs signature we constructed included 22 IRGs, and the results of GSEA indicated that some immune-related biological processes and signal pathways were enriched in high-risk patients. Such as, activation of innate immune response, MAPK signalling pathway and mTOR signalling pathway, etc. Innate immunity is a part of the HCC tumor microenvironment, which can suppress and promote cancer. For example, dendritic cells, neutrophils, and macrophages can promote the occurrence of HCC, while natural killer cells and natural killer T cells can inhibit the development of HCC (Ruf et al., 2021). The activation of MAPK signalling pathway is closely related to the development of tumors, and it is activated in about $50 \%$ of patients with early HCC and almost all patients with advanced HCC (Neuzillet et al., 2014). Excessive activation of mTOR promotes the development of tumors, and affects the immune regulation involved in the differentiation of immune cells, and plays an important role in tumor metabolism (Zou et al., 2020). There may be a link between aberrant activation of these pathways for the low overall survival of high-risk patients. We further studied the relationship between tumor immune infiltration and the IRGPs signature. In the high-risk patients of the TCGA and ICGC cohorts, aDCs, Macrophages, TH2 cells, and Treg infiltration increased. Studies have shown that the increase of regulatory DCs can promote the increase of Treg in liver cancer (Cheng et al., 2016). Treg and Macrophages, which are less infiltration in HCC, have a good prognosis (Zhu et al., 2008; Zhou et al., 2016). The increase of TH2 cells is associated with poor prognosis of HCC (Zhu et al., 2008; Zhou et al., 2010). In this study, in the TCGA and ICGC cohorts, the infiltration of Neutrophils and NK cells in high-risk patients was reduced, while the prognosis of the high-risk patients was worse than that of the low-risk patients. Studies have pointed out that neutrophils can inhibit the development of cancer (Souto et al., 2011). NK cells infiltrate less in advanced HCC and are associated with poor 


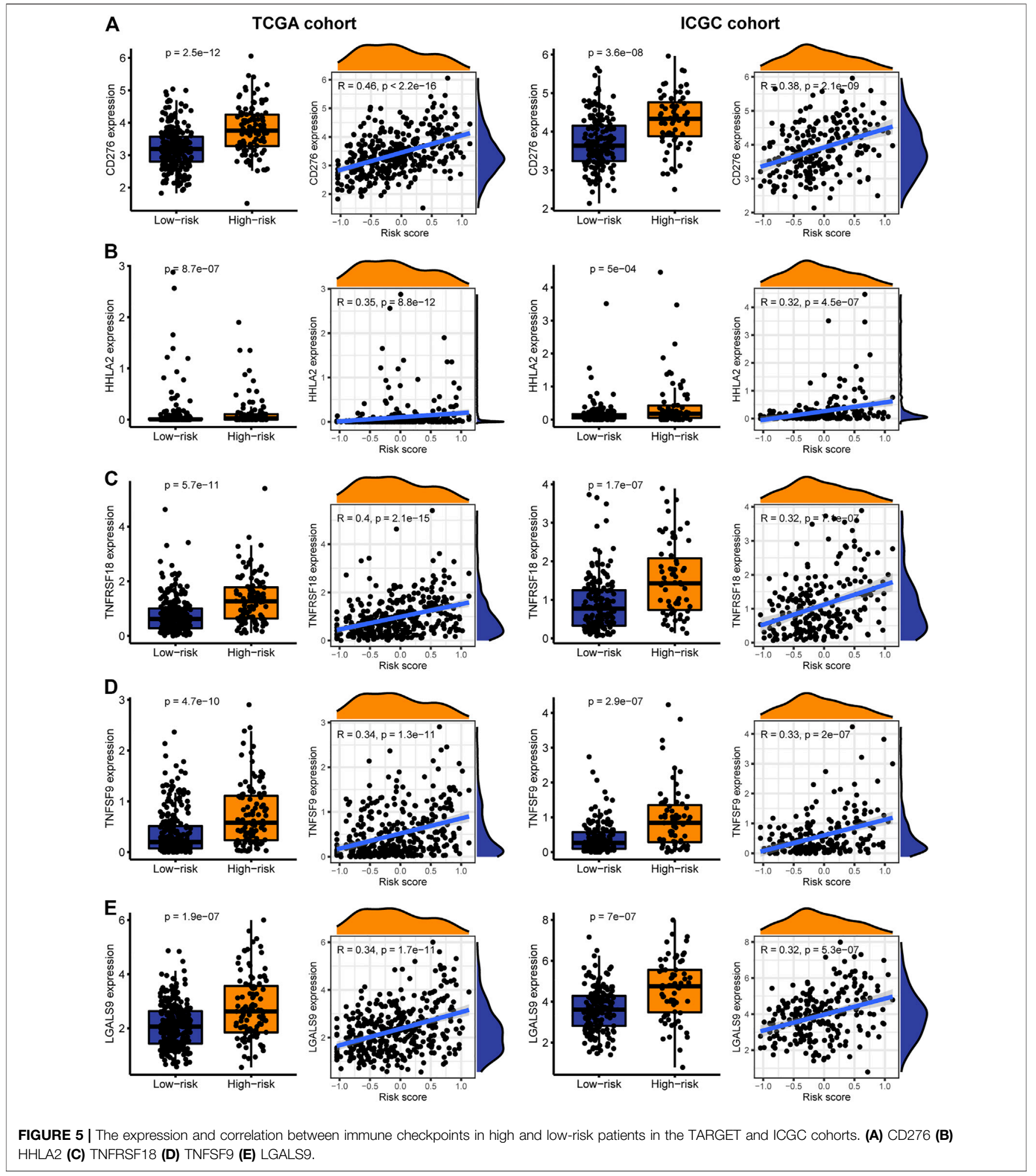

prognosis (Wu et al., 2013). These results are consistent with our research results. In the high-risk patients in the TCGA and ICGC cohorts, the type II IFN reactivity decreased, while the MHC class I activity increased. Type II IFN is mainly produced by activated NK cells (Perussia, 1991), and it plays an important role in regulating the tumor immune environment. In our study, the NK cell invasion and type II IFN reactivity in the high-risk patients were reduced, and the prognosis of the high-risk patients was poor, which was consistent with the above study results. The level of MHC class I is elevated in patients with 
A Risk 官 low 官 high

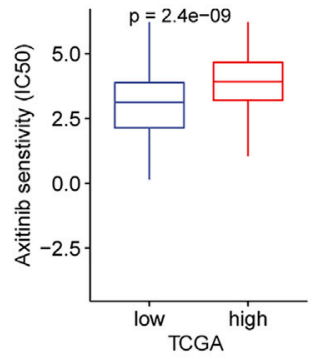

C Risk $\boxminus$ low $\boxminus$ high

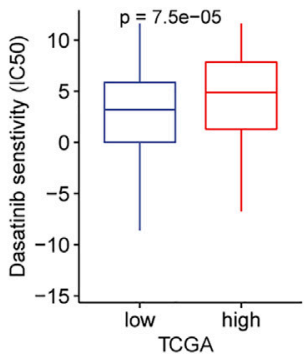

E

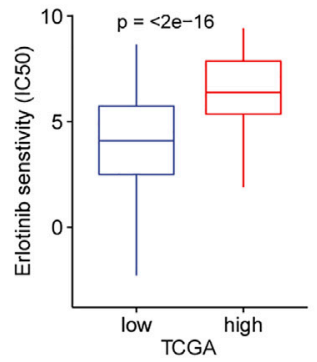

G Risk $\boxminus$ low $\boxminus$ high

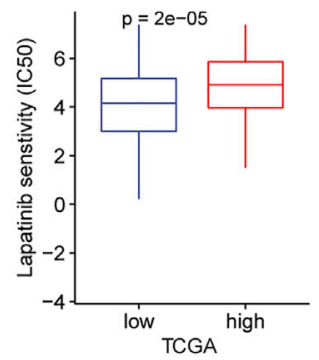

Risk 追 low 追 high

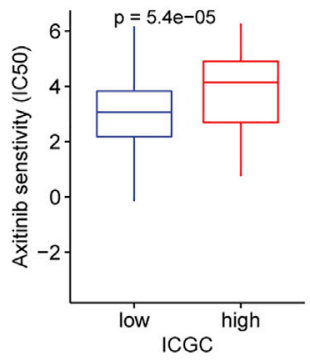

Risk $\boxminus$ low $\boxminus$ high

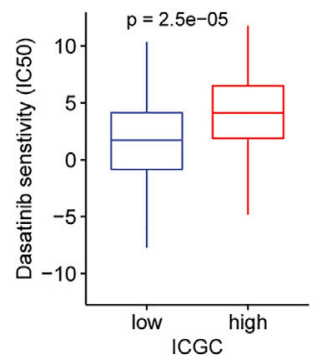

Risk 追 low 白 high

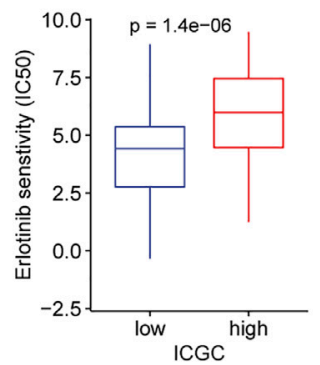

Risk $\boxminus$ low $\boxminus$ high

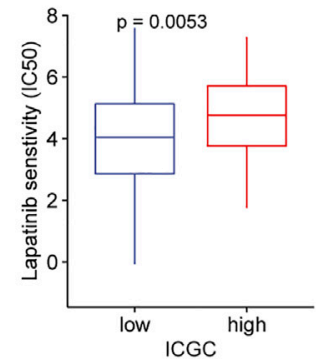

B Risk 追 low 追 high

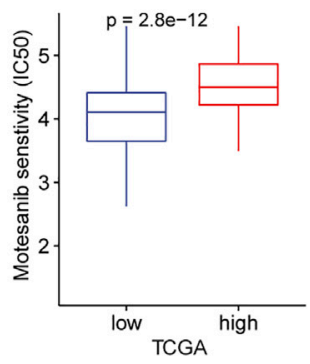

D Risk $\boxminus$ low $\boxminus$ high

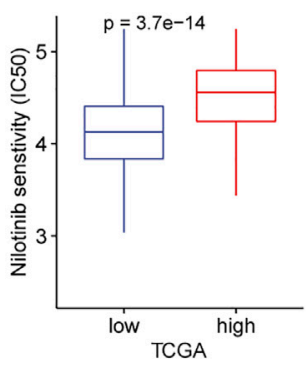

F Risk 追 low 白 high

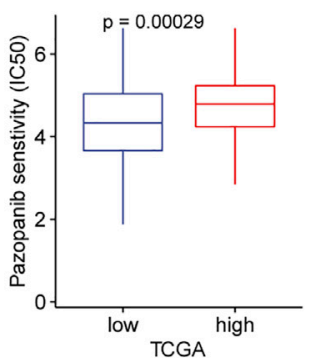

H Risk $\boxminus$ low $\boxminus$ high

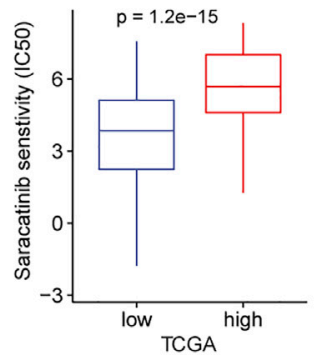

Risk 户 low 户 high

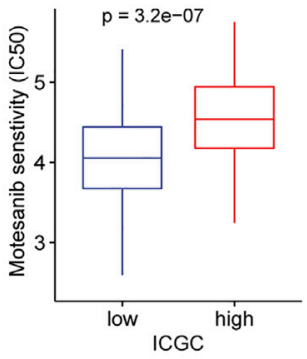

Risk $\boxminus$ low $\boxminus$ high

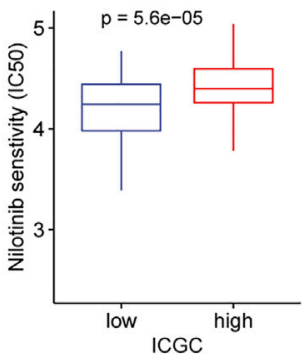

Risk 白 low 官 high

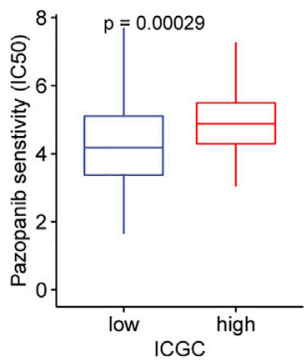

Risk $\boxminus$ low $\boxminus$ high

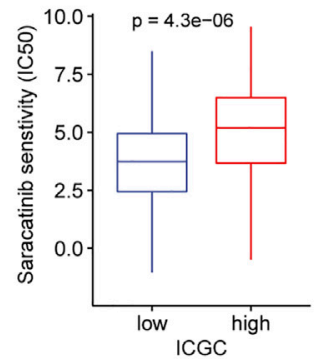

FIGURE 6 | In the TARGET and ICGC cohorts, high and low-risk patients and targeted drug sensitivity. (A) Axitinib (B) Motesanib (C) Dasatinib (D) Nilotinib (E) Erlotinib (F) Pazopanib (G) Lapatinib (H) Saracatinib.

advanced HCC, and it may negatively regulate innate immunity and adaptive immunity to cause tumor escape mechanisms to occur (Jinushi et al., 2005). According to the above findings, dysregulation of the immune microenvironment may account for the survival differences between the IRGPs signature subgroups.

Among the immune checkpoint markers, CD276, HHLA2, TNFRSF18, TNFSF9, and LGALS9 were highly expressed in high- risk patients, indicating differences in responsiveness to these immune checkpoint treatments among patients grouped by this signature. CD276 plays an important role in innate immunity and $\mathrm{T}$ cell-mediated adaptive immunity, which is highly expressed in HCC and other cancers and is associated with poor patient prognosis. And it has great potential for immunotherapy (Picarda et al., 2016). HHLA2 can inhibit the function of CD4 
and CD8 T cells, and blocking HHLA2 can enhance the proliferation and activation of $\mathrm{T}$ cells, which is helpful for cancer immunotherapy (Zhao et al., 2013; Rieder et al., 2021). TNFRSF18, also known as glucocorticoid-induced TNFR-related protein (GITR), is a costimulatory receptor in malignant tumors. Agonistic targeting of GITR can enhance the anti-tumor response of TIL derived from HCC patients (van Beek et al., 2019). TNFSF9, also known as 4-1BBL, is expressed on active $\mathrm{T}$ cells and antigen cells. 4-1BBL targeted immunotherapy has shown anti-tumor effects in the treatment of HCC (Xiao et al., 2007; Li et al., 2011). The high expression of LGALS9 is associated with the poor prognosis of many human cancers. LGALS9 preferentially kills $\mathrm{T}$ cells rather than cancer cells may assist cancer immune escape (Yang et al., 2021).

Several tyrosine kinase inhibitors have been used as first or second-line agents in the treatment of liver cancer, but one challenge is the lack of reliable biomarkers to identify patients who benefit from these treatments (Qin et al., 2019). Interestingly, we found that low-risk patients are more sensitive to Axitinib, Motesanib, Dasatinib, Nilotinib, Erlotinib, Pazopanib, Lapatinib, and Saracatinib. These findings provide effective treatment strategies for patients stratified by the IRGPs signature.

Although we used two independent data sets to rigorously verify the signature we proposed, our research has certain limitations. First of all, our study only carried out retrospective research and lacked prospective research. Second, our study only includes immune-related genes, and important prognostic genes in HCC may have been deleted. Finally, the relationship between the IRGPs signature and immune infiltration will be verified in follow-up studies.

In conclusion, the proposed IRGPs signature can accurately predict the prognosis of HCC patients and guide clinicians to

\section{REFERENCES}

Beek, A. A., Zhou, G., Doukas, M., Boor, P. P. C., Noordam, L., Mancham, S., et al. (2019). GITR Ligation Enhances Functionality of Tumor-Infiltrating T Cells in Hepatocellular Carcinoma. Int. J. Cancer 145 (4), 1111-1124. doi:10.1002/ijc.32181

Bhattacharya, S., Dunn, P., Thomas, C. G., Smith, B., Schaefer, H., Chen, J., et al. (2018). ImmPort, toward Repurposing of Open Access Immunological Assay Data for Translational and Clinical Research. Sci. Data 5, 180015. doi:10.1038/ sdata.2018.15

Binnewies, M., Roberts, E. W., Kersten, K., Chan, V., Fearon, D. F., Merad, M., et al. (2018). Understanding the Tumor Immune Microenvironment (TIME) for Effective Therapy. Nat. Med. 24 (5), 541-550. doi:10.1038/s41591-018-0014-x

Cariani, E., and Missale, G. (2019). Immune Landscape of Hepatocellular Carcinoma Microenvironment: Implications for Prognosis and Therapeutic Applications. Liver Int. 39 (9), 1608-1621. doi:10.1111/liv.14192

Cheng, J.-T., Deng, Y.-N., Yi, H.-M., Wang, G.-Y., Fu, B.-S., Chen, W.-J., et al. (2016). Hepatic Carcinoma-Associated Fibroblasts Induce Ido-producing Regulatory Dendritic Cells through IL-6-mediated STAT3 Activation. Oncogenesis 5 (2), e198. doi:10.1038/oncsis.2016.7

Chew, V., Lai, L., Pan, L., Lim, C. J., Li, J., Ong, R., et al. (2017). Delineation of an Immunosuppressive Gradient in Hepatocellular Carcinoma Using HighDimensional Proteomic and Transcriptomic Analyses. Proc. Natl. Acad. Sci. USA 114 (29), E5900-E5909. doi:10.1073/pnas.1706559114

Dong, L.-Q., Peng, L.-H., Ma, L.-J., Liu, D.-B., Zhang, S., Luo, S.-Z., et al. (2020). Heterogeneous Immunogenomic Features and Distinct Escape Mechanisms in make specific treatment decisions. It also provides the relationship between this signature and the responsiveness of immune checkpoints and targeted drugs. These results will be beneficial to the effective treatment of HCC patients. Prospective studies are required to further verify its accuracy.

\section{DATA AVAILABILITY STATEMENT}

Publicly available datasets were analyzed in this study. This data can be found here: https://portal.gdc.cancer.gov/; https://daco. icgc.org/; https://www.immport.org/home.

\section{AUTHOR CONTRIBUTIONS}

SM and YM: conceptualization. XF and SM: software and methodology. XF and YM: writing-original draft preparation. WW: writing-review and editing. All authors have read and agreed to the published version of the article.

\section{ACKNOWLEDGMENTS}

We would like to acknowledge the TCGA and the ICGC for providing data.

\section{SUPPLEMENTARY MATERIAL}

The Supplementary Material for this article can be found online at: https://www.frontiersin.org/articles/10.3389/fmolb.2021.715728/ full\#supplementary-material

Multifocal Hepatocellular Carcinoma. J. Hepatol. 72 (5), 896-908. doi:10.1016/ j.jhep.2019.12.014

Forner, A., Reig, M., and Bruix, J. (2018). Hepatocellular Carcinoma. The Lancet 391 (10127), 1301-1314. doi:10.1016/S0140-6736(18)30010-2

Gan, X., Luo, Y., Dai, G., Lin, J., Liu, X., Zhang, X., et al. (2020). Identification of Gene Signatures for Diagnosis and Prognosis of Hepatocellular Carcinomas Patients at Early Stage. Front. Genet. 11, 857. doi:10.3389/fgene.2020.00857

Geeleher, P., Cox, N., and Huang, R. S. (2014). pRRophetic: an R Package for Prediction of Clinical Chemotherapeutic Response from Tumor Gene Expression Levels. PloS one 9 (9), e107468. doi:10.1371/journal.pone.0107468

Hänzelmann, S., Castelo, R., and Guinney, J. (2013). GSVA: Gene Set Variation Analysis for Microarray and RNA-Seq Data. BMC bioinformatics 14, 7 . doi:10.1186/1471-2105-14-7

Hong, W., Liang, L., Gu, Y., Qi, Z., Qiu, H., Yang, X., et al. (2020). Immune-Related lncRNA to Construct Novel Signature and Predict the Immune Landscape of Human Hepatocellular Carcinoma. Mol. Ther. - Nucleic Acids 22, 937-947. doi:10.1016/j.omtn.2020.10.002

Ino, Y., Yamazaki-Itoh, R., Shimada, K., Iwasaki, M., Kosuge, T., Kanai, Y., et al. (2013). Immune Cell Infiltration as an Indicator of the Immune Microenvironment of Pancreatic Cancer. Br. J. Cancer 108 (4), 914-923. doi:10.1038/bjc.2013.32

Jinushi, M., Takehara, T., Tatsumi, T., Hiramatsu, N., Sakamori, R., Yamaguchi, S., et al. (2005). Impairment of Natural Killer Cell and Dendritic Cell Functions by the Soluble Form of MHC Class I-Related Chain A in Advanced Human Hepatocellular Carcinomas. J. Hepatol. 43 (6), 1013-1020. doi:10.1016/j.jhep.2005.05.026

Kaur, H., Dhall, A., Kumar, R., and Raghava, G. P. S. (2019). Identification of Platform-Independent Diagnostic Biomarker Panel for Hepatocellular 
Carcinoma Using Large-Scale Transcriptomics Data. Front. Genet. 10, 1306. doi:10.3389/fgene.2019.01306

Kaur, H., Lathwal, A., and Raghava, G. P. S. (2021). Integrative Multi-Omics Approach for Stratification of Tumor Recurrence Risk Groups of Hepatocellular Carcinoma Patients. bioRxiv. doi:10.1101/2021.03.03.433841

Kumar, S. U., Kumar, D. T., Siva, R., Doss, C. G. P., and Zayed, H. (2019). Integrative Bioinformatics Approaches to Map Potential Novel Genes and Pathways Involved in Ovarian Cancer. Front. Bioeng. Biotechnol. 7, 391. doi:10.3389/fbioe.2019.00391

Kurebayashi, Y., Ojima, H., Tsujikawa, H., Kubota, N., Maehara, J., Abe, Y., et al. (2018). Landscape of Immune Microenvironment in Hepatocellular Carcinoma and its Additional Impact on Histological and Molecular Classification. Hepatology 68 (3), 1025-1041. doi:10.1002/hep.29904

Li, B., Cui, Y., Diehn, M., and Li, R. (2017a). Development and Validation of an Individualized Immune Prognostic Signature in Early-Stage Nonsquamous Non-Small Cell Lung Cancer. JAMA Oncol. 3 (11), 1529-1537. doi:10.1001/ jamaoncol.2017.1609

Li, B., Feng, W., Luo, O., Xu, T., Cao, Y., Wu, H., et al. (2017b). Development and Validation of a Three-Gene Prognostic Signature for Patients with Hepatocellular Carcinoma. Sci. Rep. 7 (1), 5517. doi:10.1038/s41598-017-04811-5

Li, G., Wu, X., Zhang, F., Li, X., Sun, B., Yu, Y., et al. (2011). Triple Expression of B7-1, B7-2 and 4-1BBL Enhanced Antitumor Immune Response against Mouse H22 Hepatocellular Carcinoma. J. Cancer Res. Clin. Oncol. 137 (4), 695-703. doi:10.1007/s00432-010-0905-9

Liu, M., Liu, X., Liu, S., Xiao, F., Guo, E., Qin, X., et al. (2020). Big Data-Based Identification of Multi-Gene Prognostic Signatures in Liver Cancer. Front. Oncol. 10, 847. doi:10.3389/fonc.2020.00847

Neuzillet, C., Tijeras-Raballand, A., de Mestier, L., Cros, J., Faivre, S., and Raymond, E. (2014). MEK in Cancer and Cancer Therapy. Pharmacol. Ther. 141 (2), 160-171. doi:10.1016/j.pharmthera.2013.10.001

Nishida, N., and Kudo, M. (2017). Immunological Microenvironment of Hepatocellular Carcinoma and its Clinical Implication. Oncology 92 (Suppl. 1), 40-49. doi:10.1159/000451015

Ouyang, G., Yi, B., Pan, G., and Chen, X. (2020). A Robust Twelve-Gene Signature for Prognosis Prediction of Hepatocellular Carcinoma. Cancer Cel Int 20, 207. doi:10.1186/s12935-020-01294-9

Peng, D., Wang, L., Li, H., Cai, C., Tan, Y., Xu, B., et al. (2019). An Immune Infiltration Signature to Predict the Overall Survival of Patients with colon Cancer. IUBMB life 71 (11), 1760-1770. doi:10.1002/iub.2124

Perussia, B. (1991). Lymphokine-Activated Killer Cells, Natural Killer Cells and Cytokines. Curr. Opin. Immunol. 3 (1), 49-55. doi:10.1016/0952-7915(91)90076-d

Picarda, E., Ohaegbulam, K. C., and Zang, X. (2016). Molecular Pathways: Targeting B7-H3 (CD276) for Human Cancer Immunotherapy. Clin. Cancer Res. 22 (14), 3425-3431. doi:10.1158/1078-0432.CCR-15-2428

Prieto, J., Melero, I., and Sangro, B. (2015). Immunological Landscape and Immunotherapy of Hepatocellular Carcinoma. Nat. Rev. Gastroenterol. Hepatol. 12 (12), 681-700. doi:10.1038/nrgastro.2015.173

Qin, S., Li, A., Yi, M., Yu, S., Zhang, M., and Wu, K. (2019). Recent Advances on Anti-Angiogenesis Receptor Tyrosine Kinase Inhibitors in Cancer Therapy. J. Hematol. Oncol. 12 (1), 27. doi:10.1186/s13045-019-0718-5

Rieder, S. A., Wang, J., White, N., Qadri, A., Menard, C., Stephens, G., et al. (2021). B7-H7 (HHLA2) Inhibits T-Cell Activation and Proliferation in the Presence of TCR and CD28 Signaling. Cell Mol Immunol 18 (6), 1503-1511. doi:10.1038/ s41423-020-0361-7

Ritchie, M. E., Phipson, B., Wu, D., Hu, Y., Law, C. W., Shi, W., et al. (2015). Limma powers Differential Expression Analyses for RNA-Sequencing and Microarray Studies. Nucleic Acids Res. 43 (7), e47. doi:10.1093/nar/gkv007

Ruf, B., Heinrich, B., and Greten, T. F. (2021). Immunobiology and Immunotherapy of HCC: Spotlight on Innate and Innate-Like Immune Cells. Cel Mol Immunol 18 (1), 112-127. doi:10.1038/s41423-020-00572-w

Siegel, R. L., Miller, K. D., and Jemal, A. (2019). Cancer Statistics, 2019. CA A. Cancer J. Clin. 69 (1), 7-34. doi:10.3322/caac.21551

Souto, J. C., Vila, L., and Brú, A. (2011). Polymorphonuclear Neutrophils and Cancer: Intense and Sustained Neutrophilia as a Treatment against Solid Tumors. Med. Res. Rev. 31 (3), 311-363. doi:10.1002/med.20185

Udhaya Kumar, S., Madhana Priya, N., Thirumal Kumar, D., Anu Preethi, V., Kumar, V., Nagarajan, D., et al. (2021). An Integrative Analysis to Distinguish between Emphysema (EML) and Alpha-1 Antitrypsin Deficiency-Related
Emphysema (ADL)-A Systems Biology Approach. Adv. Protein Chem. Struct. Biol. 127, 315-342. doi:10.1016/bs.apcsb.2021.02.004

Udhaya Kumar, S., Thirumal Kumar, D., Bithia, R., Sankar, S., Magesh, R., Sidenna, M., et al. (2020). Analysis of Differentially Expressed Genes and Molecular Pathways in Familial Hypercholesterolemia Involved in Atherosclerosis: A Systematic and Bioinformatics Approach. Front. Genet. 11, 734. doi:10.3389/ fgene.2020.00734

Villanueva, A. (2019). Hepatocellular Carcinoma. N. Engl. J. Med. 380 (15), 1450-1462. doi:10.1056/NEJMra1713263

Wu, Y., Kuang, D.-M., Pan, W.-D., Wan, Y.-L., Lao, X.-M., Wang, D., et al. (2013). Monocyte/macrophage-elicited Natural Killer Cell Dysfunction in Hepatocellular Carcinoma Is Mediated by CD48/2B4 Interactions. Hepatology 57 (3), 1107-1116. doi:10.1002/hep.26192

Xiao, H., Huang, B., Yuan, Y., Li, D., Han, L.-F., Liu, Y., et al. (2007). Soluble PD-1 Facilitates 4-1bbl-Triggered Antitumor Immunity against Murine $\mathrm{H} 22$ Hepatocarcinoma In Vivo. Clin. Cancer Res. 13 (6), 1823-1830. doi:10.1158/ 1078-0432.CCR-06-2154

Yang, J. D., Hainaut, P., Gores, G. J., Amadou, A., Plymoth, A., and Roberts, L. R. (2019). A Global View of Hepatocellular Carcinoma: Trends, Risk, Prevention and Management. Nat. Rev. Gastroenterol. Hepatol. 16 (10), 589-604. doi:10.1038/s41575-019-0186-y

Yang, R., Sun, L., Li, C.-F., Wang, Y.-H., Yao, J., Li, H., et al. (2021). Galectin-9 Interacts with PD-1 and TIM-3 to Regulate T Cell Death and Is a Target for Cancer Immunotherapy. Nat. Commun. 12 (1), 832. doi:10.1038/s41467-021-21099-2

Zhao, R., Chinai, J. M., Buhl, S., Scandiuzzi, L., Ray, A., Jeon, H., et al. (2013). HHLA2 Is a Member of the B7 Family and Inhibits Human CD4 and CD8 T-Cell Function. Proc. Natl. Acad. Sci. 110 (24), 9879-9884. doi:10.1073/pnas.1303524110

Zheng, C., Zheng, L., Yoo, J.-K., Guo, H., Zhang, Y., Guo, X., et al. (2017). Landscape of Infiltrating T Cells in Liver Cancer Revealed by Single-Cell Sequencing. Cell 169 (7), 1342-1356. doi:10.1016/j.cell.2017.05.035

Zhou, H., Huang, H., Shi, J., Zhao, Y., Dong, Q., Jia, H., et al. (2010). Prognostic Value of Interleukin 2 and Interleukin 15 in Peritumoral Hepatic Tissues for Patients with Hepatitis B-Related Hepatocellular Carcinoma after Curative Resection. Gut 59 (12), 1699-1708. doi:10.1136/gut.2010.218404

Zhou, S.-L., Zhou, Z.-J., Hu, Z.-Q., Huang, X.-W., Wang, Z., Chen, E.-B., et al. (2016). Tumor-Associated Neutrophils Recruit Macrophages and T-Regulatory Cells to Promote Progression of Hepatocellular Carcinoma and Resistance to Sorafenib. Gastroenterology $150 \quad$ (7), 1646-1658. doi:10.1053/ j.gastro.2016.02.040

Zhou, T., Cai, Z., Ma, N., Xie, W., Gao, C., Huang, M., et al. (2020). A Novel TenGene Signature Predicting Prognosis in Hepatocellular Carcinoma. Front. Cel Dev. Biol. 8, 629. doi:10.3389/fcell.2020.00629

Zhu, X.-D., Zhang, J.-B., Zhuang, P.-Y., Zhu, H.-G., Zhang, W., Xiong, Y.-Q., et al. (2008). High Expression of Macrophage Colony-Stimulating Factor in Peritumoral Liver Tissue Is Associated with Poor Survival after Curative Resection of Hepatocellular Carcinoma. J. Clin. Oncol. 26 (16), 2707-2716. doi:10.1200/jco.2007.15.6521

Zou, Z., Tao, T., Li, H., and Zhu, X. (2020). mTOR Signaling Pathway and mTOR Inhibitors in Cancer: Progress and Challenges. Cell Biosci 10, 31. doi:10.1186/ s13578-020-00396-1

Conflict of Interest: The authors declare that the research was conducted in the absence of any commercial or financial relationships that could be construed as a potential conflict of interest.

Publisher's Note: All claims expressed in this article are solely those of the authors and do not necessarily represent those of their affiliated organizations, or those of the publisher, the editors and the reviewers. Any product that may be evaluated in this article, or claim that may be made by its manufacturer, is not guaranteed or endorsed by the publisher.

Copyright $(2) 2021 \mathrm{Feng}, \mathrm{Mu}, \mathrm{Ma}$ and Wang. This is an open-access article distributed under the terms of the Creative Commons Attribution License (CC BY). The use, distribution or reproduction in other forums is permitted, provided the original author(s) and the copyright owner(s) are credited and that the original publication in this journal is cited, in accordance with accepted academic practice. No use, distribution or reproduction is permitted which does not comply with these terms. 